\title{
Membrane chemical stability and seed longevity
}

\author{
Elena A. Golovina $\cdot$ Henk Van As • \\ Folkert A. Hoekstra
}

Received: 15 May 2009/Revised: 3 September 2009/Accepted: 9 September 2009/Published online: 13 October 2009

(C) The Author(s) 2009. This article is published with open access at Springerlink.com

\begin{abstract}
Here, we investigate the relationships between the chemical stability of the membrane surface and seed longevity. Dry embryos of long-lived tomato and shortlived onion seeds were labeled with 5-doxyl-stearic acid (5-DS). Temperature-induced loss of the electron spin resonance signal caused by chemical conversion of 5-DS to nonparamagnetic species was used to characterize the membrane surface chemical stability. No difference was found between temperature plots of 5-DS signal intensity in dry onion and tomato below $345 \mathrm{~K}$. Above this temperature, the 5-DS signal remained unchanged in tomato embryos and irreversibly disappeared in onion seeds. The role of the physical state and chemical status of the membrane environment in the chemical stability of membrane surfaces was estimated for model systems containing 1-palmitoyl-2-oleoyl-sn-glycero-3-phosphocholine (POPC) dried alone or in the presence of trehalose or glucose. Fourier transform infrared spectroscopy was used to follow temperature-induced structural changes in dry POPC. Spinlabel technique was used to relate the chemical stability of 5-DS with the dynamic properties of the bilayer and 5-DS motion behavior. In all the models, the decrease in 5-DS signal intensity was always observed above $T_{\mathrm{m}}$ for the membrane surface. The 5-DS signal was irreversibly lost at
\end{abstract}

The more you see: spectroscopy in molecular biophysics.

E. A. Golovina $(\bowtie)$ · F. A. Hoekstra

Laboratory of Plant Physiology, Wageningen University,

Arboretumlaan 4, 6703 BD Wageningen, The Netherlands

e-mail: elena.golovina@wur.nl

E. A. Golovina - H. Van As

Laboratory of Biophysics and Wageningen NMR Centre,

Wageningen University, Dreijenlaan 3,

6703 HA Wageningen, The Netherlands high temperature when dry POPC was embedded in a glucose matrix. The loss of 5-DS signal was moderate when POPC was dried alone or in the presence of trehalose. Comparison of model and in vivo data shows that the differences in longevity between onion and tomato seeds are caused by differences in the chemical status of the membrane surface rather than the degree of its immobilization.

Keywords Dry systems - Membrane physical state . Seed longevity $\cdot$ Spin label chemical conversion
Abbreviations
5-DS 5-DOXYL-stearic acid
ESR Electron spin resonance
FT-IR Fourier transform infrared spectroscopy
P50 Half-viability period
POPC 1-palmitoyl-2-oleoyl-sn-glycero-3 phosphocholine
$T_{\mathrm{m}} \quad$ Membrane phase transition temperature

\section{Introduction}

Seeds of many species are desiccation tolerant, i.e., they can be dried to the air-dry state and resume normal functioning on rehydration. Dry seeds of most species are able to survive much longer than the next growing season. This ability of seeds is used in seed industry and in seed banks for dry storage. However, deterioration of seeds in dry storage is inevitable. Seed storability (or longevity) can be quantified in terms of the half-viability period, P50 (Roberts 1973; Ellis and Roberts 1980). Seeds of major 
crops are tabulated according to their P50 into three classes: short-lived, long-lived, and intermediate (Copeland and McDonald 2001). Nevertheless, the exact longevity of seeds in storage remains uncertain because it can be modified by environment. This makes the ability to predict seed longevity extremely important, since loss of seed quality would be able to be anticipated (Copeland and McDonald 2001).

Different approaches have been developed to assess seed storage time without loss of viability. The obvious way to predict seed storage behavior is the germination test after exposing seeds to adverse conditions, which accelerates deterioration (Delouche and Baskin 1973). Artificial aging and controlled deterioration tests are widely used to predict seed storage behavior. However, because of predictive limitations (Hay et al. 2003; Tang et al. 2000) and length of time required for aging tests, there is continuing interest in discovering some biochemical or biophysical characteristics of seeds that can be used to predict their longevity. The search for such characteristics is based on understanding the molecular mechanisms of seed longevity.

Seed deterioration at the molecular level involves many chemical reactions that can occur in a dry state (Priestley 1986). Damage accumulates with time because repair is not possible in dry biological systems. Molecular mechanisms of seed longevity include all factors that can slow down the rate of chemical reactions in a dry state and, thus, increase time for critical accumulation of damage. Auto-oxidation is considered the main chemical reaction causing seed deterioration (Priestley 1986; Hendry 1993). Factors that can decrease the rate of auto-oxidation can conditionally be divided into two classes-physical and chemical. Physical factors include molecular immobilization at all levels, which limits molecular diffusion and capacity for chemical reactions. Chemical factors include susceptibility to oxidation, formation of reactive oxygen species, and presence and efficiency of antioxidants. The role of physical and chemical factors in seed longevity has been extensively studied.

Glassy state has been studied in relation to seed longevity (Leopold et al. 1994; Sun 1997; Narayana Murthy et al. 2003). Buitink conducted comprehensive research on causal relationships between molecular immobilization in cytoplasmic glasses and seed longevity (Buitink et al. 1998, 2000). However, regression lines obtained for different species do not match each other (Buitink et al. 2000). It seems that molecular immobilization in a dry cytoplasm can be a marker of seed longevity, only when other factors of seed longevity are identical, i.e., within the same specimen at different water contents or temperatures. Therefore, the degree of molecular immobilization cannot be used as a general predictor of longevity of dry biological systems. Indeed, broad studies of many anhydrobiotes show that there is no correlation between the glass transition temperature of biological glasses and the longevity of dry organisms (Hoekstra 2005).

Obviously, not only the physical state of dry organisms, but also their chemical status has to be taken into consideration. There is a considerable body of literature showing that downregulation of metabolism in desiccation-tolerant organisms prevents accumulation of reactive oxygen species during drying (reviewed in Buitink et al. 2002). The role of antioxidants in seed longevity is reviewed by Bailly (2004) and Beckman and Ames (1997). Hoekstra (2005) has shown a linear correlation between the double-bond index of polar lipids (average number of double bonds per acyl chain) and the longevity of 21 different dry organisms. This indicates that membrane susceptibility to oxidation is an important factor in seed longevity. Therefore, all these factors are clearly involved in seed longevity. However, the predictive value of each of these factors, taken alone, is low because of the multi-component character of seed protection in dry storage.

In this paper we propose another approach, which includes all of the above-mentioned factors and therefore might be used to predict seed longevity. The approach is based on monitoring the chemical stability of the membrane surface environment. Membranes are selected for the following reasons. First, membranes are the most probable site of primary damage in aging (Benson 1990; Priestley 1986; Wilson and McDonald 1986). Second, membrane chemical modification (of both lipids and proteins) is most critical for cell functioning. Membrane chemical stability is determined by the degree of membrane protection against chemical modification and involves membrane immobilization, and the chemical status of membranes (susceptibility to chemical modification) and the nearby environment (accessibility of chemically active species and antioxidants). The role of different factors in membrane deterioration might be different in different systems. However, membrane chemical stability will reflect the total resistance of the membrane environment to changes without respect to exact mechanisms of membrane protection.

Membrane spin probe 5-DOXYL stearic acid (5-DS) is a commonly used nitroxide to characterize the degree of ordering at the membrane surface. However, 5-DS also can be used to get information on chemical reactivity of binding sites (Stier and Sackmann 1973). It is well established that nitroxide spin probes can be reduced or oxidized to the corresponding nonparamagnetic species (Swartz 1987, 1990). This causes loss of ESR signal and, therefore, is often considered a problem for in vivo applications of the spin label technique (discussed in Marsh 1981; Morse 1985). However, nitroxide reduction/oxidation can also provide unique information about systems under study. The predominant reaction of nitroxides in living cells is their 
reversible reduction to hydroxylamines (Chen and Swartz 1988, 1989; Sentjurc et al. 1990). Reduction of nitroxides by living cells or isolated membranes was first described in the early 1970s (Kaplan et al. 1973; Stier and Reit 1971). Swartz (1987) proposed using nitroxides to measure redox metabolism in cells and tissues. Because oxidation of hydroxylamines to nitroxides depends on oxygen concentration inside the cell, the rate of re-oxidation of hydroxylamines allows the measurements of local oxygen concentrations (Bobko et al. 2007; Chen and Swartz 1988; Chen et al. 1989). An extensive review of the metabolism of nitroxides was published in 1995 (Kocherginsky et al. 1995). Typically, a nitroxide is administered to metabolizing cells, and the rate of signal disappearance from the sample is followed over time. The rate of signal disappearance depends on rate constants for the reduction of the nitroxide $\left(K_{\mathrm{R}}\right)$ and on the rate of re-oxidation of the hydroxylamine, $K_{\mathrm{O}} . K_{\mathrm{R}}$ is the sum of rate constants for enzymatic and chemical reduction, and $K_{\mathrm{O}}$ is the sum of the rate constants of enzymatic oxidation and auto-oxidation (Chen and Swartz 1988). As far as membrane spin labels is concerned, a systematic study of reduction of doxylstearates in labeled cells has shown that 5-DS and the reducing equivalents are in frequent contact. From this observation, it has become evident that the source of reducing equivalents for 5-DS is near the surface of the membrane (Chen et al. 1988).

In this paper we propose to use 5-DS to detect membrane chemical stability in a dry state. Although nothing is known about the chemical reactions of nitroxides in a dry state, the decrease in signal intensity of 5-DS is the result of chemical reduction and/or oxidation of spin label to nonparamagnetic species. Chemical reactions in dry systems are very slow and can hardly be studied within reasonable experimental time at room temperature. However, the increase in temperature might enhance the probability of chemical reactions of nitroxides with their environment and, thus, decrease signal intensity. Chemical thermostability of 5-DS might include the same factors that determine chemical stability of the membrane surface. Membrane resistance to temperature-induced chemical modification of 5-DS is intimately linked to time-induced changes. Subjecting samples to elevated temperatures for specific periods of time is often used to simulate the effects of real-time aging and provides data that allows the prediction of the shelf-life of dry food or medical products.

As a first step to test the validity of this approach, we conducted a comparative study of tomato (Lycopersicon esculentum) and onion (Allium cepa) seeds, which have considerable differences in longevity. According to Priestley et al. (1985) P50 $=5.43$ years for onion and 24.52 years for tomato seeds. Dry embryos of these seeds were labeled in vivo with 5-DS. The results showed that chemical stability of 5-DS was much higher in tomato than in onion dry embryos. These experiments prove that the proposed approach can potentially be used to characterize seed longevity. We also conducted model experiments to find the influence of physical and chemical factors on chemical thermostability at the membrane surface. We studied the properties of the POPC (1-palmitoyl-2-oleoyl$s n$-glycero-3 phosphocholine) bilayer dried alone and in the presence of glucose or trehalose. Dynamic properties of dry POPC bilayer were studied using Fourier transform infrared spectroscopy (FT-IR). Chemical stability at the bilayer interface was concluded from the temperature plots of ESR signal intensity of dry POPC bilayer labeled with 5-DS. We have shown that although the thermotropic behavior of a dry bilayer is different in different model systems, the disappearance of the 5-DS signal always coincided with the melting of the membrane surface; the degree and the reversibility of signal loss depended on the redox potential of the environment.

\section{Experimental}

\section{Chemicals}

The lipid 1-palmitoyl-2-oleoyl-sn-glycero-3 phosphocholine (POPC, Avanti Polar Lipids, Alabaster, AL, USA), sugars $\beta$-D-glucose and trehalose (Pfahnstiel, Waukegan, IL, USA), and spin label 5-DOXYL-stearic acid (5-DS, Sigma, St. Louis, MO, USA) were used as received.

\section{Plant materials}

Viable tomato (Lycopersicon esculentum, cv Melville Castle, UK, CGN 14444) and onion (Allium cepa cv White Spanish Purnong Strain, Australia, CGN 24143) seeds were obtained from the Centre for Genetic Resources, the Netherlands (CGN).

\section{FT-IR experiments}

POPC (2 mg) dissolved in chloroform was placed in a slight stream of nitrogen at room temperature. Remaining traces of solvent were removed under vacuum overnight. For FT-IR experiments, POPC was dispersed in $0.5 \mathrm{ml}$ of water. Hydration was facilitated by five freeze-thaw cycles and vortexing. The solution was further sonicated in a round ultrasonic bath at room temperature for $1 \mathrm{~min}$. POPC dispersions in sugars were prepared by using $0.5 \mathrm{ml}$ of respective solutions instead of water. The molar ratios were POPC: glucose $=1: 21$ and POPC:trehalose $=1: 11$. Then, $5 \mu \mathrm{l}$ of the sample was dried on a circular $\mathrm{CaF}_{2}(13 \times 2 \mathrm{~mm}$ diameter) window in a cabinet continuously purged with dry 
air $(\mathrm{RH} \sim 3 \%)$ at room temperature for at least $24 \mathrm{~h}$. Subsequently, each sample was hermetically sealed by applying a second IR-window using a rubber O-ring in between and mounted on a temperature-controlled brass cell. These procedures were performed in the above described cabinet to prevent possible rehydration of dry samples.

IR spectra were recorded on a Perkin-Elmer 1725 FT-IR spectrometer (Perkin-Elmer, Beaconsfield, UK) equipped with an external beam facility to which a Perkin-Elmer IR microscope was attached. The microscope was equipped with a narrow-band liquid nitrogen-cooled mercury/cadmium/telluride detector. Temperature of the brass cell was regulated by a computer-controlled device that activated a liquid nitrogen pump in conjunction with a power supply for heating of the cell (Wolkers et al. 1998). The sample temperature was recorded separately using a PT-100 element that was located very close to the sample windows. Spectra were recorded every minute at steps of $1.5^{\circ} \mathrm{C}$, starting with the lowest temperature. The optical bench was purged with dry $\mathrm{CO}_{2}$-free air (Balston, Maidstone, UK) at a flow rate of $25 \mathrm{l} / \mathrm{min}$. The acquisition parameters were $4 \mathrm{~cm}^{-1}$ resolution, 32 co-added interferograms, $2 \mathrm{~cm} \mathrm{~s}^{-1}$ moving mirror speed, and 3,600 to $1,000 \mathrm{~cm}^{-1}$ wavenumber range. Spectral analysis was carried out using Spectrum version 2.00 (Perkin-Elmer).

\section{ESR experiments}

Spin labeling of POPC dispersions was carried out by adding $1 \mathrm{~mol} \%$ of 5-DS to the initial chloroform solution of POPC. The subsequent steps of sample preparation were as described above. POPC dispersion in water or in solutions of sugars was spread over chemically inert glass beads (80-110 $\mu \mathrm{m}$ diameter) on a glass slide and allowed to dry overnight in a stream of dry air $(\sim 3 \% \mathrm{RH})$. The dried material was transferred to 2-mm capillaries in a dryair box $(\sim 3 \% \mathrm{RH})$ and then flame-sealed.

Onion and tomato seeds have similar morphology - the embryos are curved and surrounded by endosperm and seed coat. Because it was practically impossible to isolate embryos from dry seeds, seeds were rehydrated for $6 \mathrm{~h}$. After rehydration, embryos were isolated and fast-dried in the dry-air box at $\sim 3 \%$ RH. After 3 days of equilibration at $\mathrm{RH} \sim 3 \%$, embryos were labeled with 5-DS in $1 \mathrm{mM}$ solution of 5-DS in hexane for 1 day. After labeling, dry samples were blotted with filter paper and placed at $\mathrm{RH}$ $\sim 3 \%$ for hexane evaporation for 1 day. After that, dry embryos were transferred to 2-mm capillaries in the dry-air box and flame-sealed.

The capillary with the sample was placed in an ESR quartz tube for spectrum recording. ESR spectra were recorded with an X-band ESR spectrometer (Elexsys model E 500; Bruker Analytik, Rheinstetten, Germany) equipped with a temperature unit using regular air within the temperature range $300-400 \mathrm{~K}$ and liquid $\mathrm{N}_{2}$ at below room temperature. The spectra were recorded at $5^{\circ}$ increments with equilibration for $1 \mathrm{~min}$ at each temperature. The scan range was $100 \mathrm{G}$ for all spectra. To prevent over-modulation and saturation of ESR signal, the modulation amplitude was $2.5 \mathrm{G}$ or less, and the microwave power was limited to $5 \mathrm{~mW}$.

All ESR and FT-IR experiments on model systems were conducted on the same samples prepared under the same conditions. Each model experiment was repeated at least twice, and the results of the single experiments are presented.

\section{Results}

5-DS ordering and chemical stability in dry embryos of tomato and onion seeds

ESR spectra of 5-DS in dry onion embryos at $T<290 \mathrm{~K}$ are solid-like (Fig. 1). At $T>290 \mathrm{~K}$ the second fluid-like component appears (arrow in Fig. 1) and becomes dominant at $T>340 \mathrm{~K}$. Similar changes of the spectral shape with temperature are observed for 5-DS in dry tomato embryos (spectra not shown). The degree of ordering of 5 -DS is characterized by the distance $\left(2 \mathrm{~A}_{\max }\right)$ between two

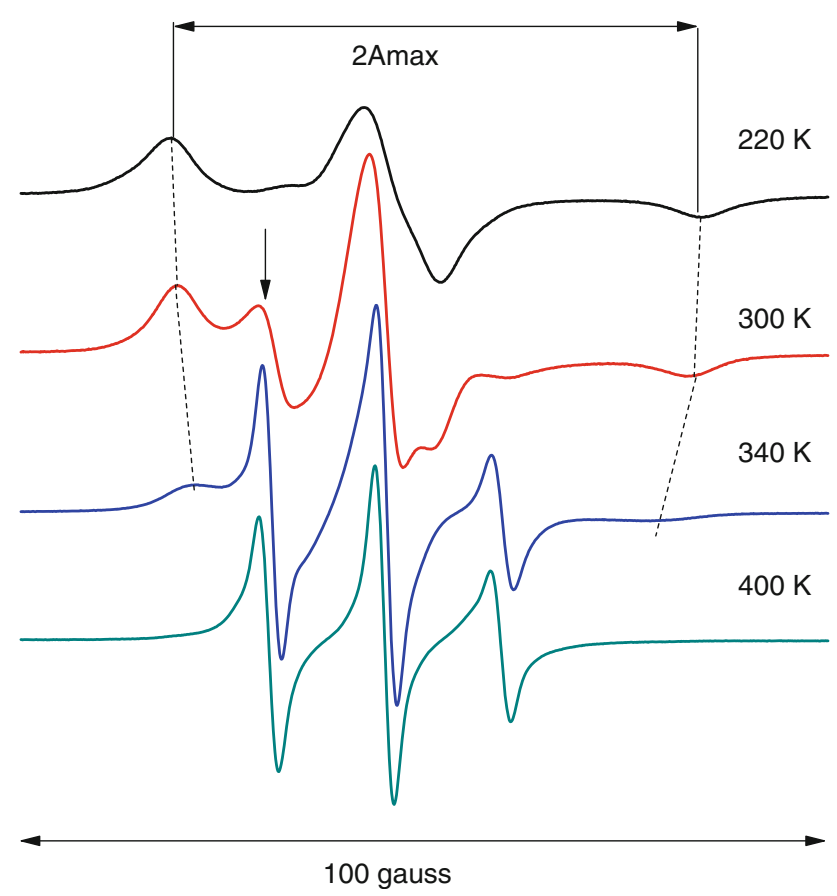

Fig. 1 ESR spectra of 5-DS in dry onion embryos at different temperatures. The position of the low-field (lefthand) line of the fluid component is indicated by the arrow. The spectra are recorded within the range of the magnetic field strength of 100 gauss 
outer hyperfine extremes of the solid-like ESR spectra (Fig. 1). This distance decreases with temperature due to disordering of 5-DS in membranes. The temperature plots of $2 \mathrm{~A}_{\max }$ for onion and tomato embryos are similar and can be approximated linearly within the $220-285 \mathrm{~K}$ interval when plotted in Arrhenius coordinates (Fig. 2a, adjusted $R^{2}=0.99256$ for onion and 0.99181 for tomato embryos). Above $345 \mathrm{~K}, 2 \mathrm{~A}_{\max }$ cannot be determined (Fig. 1) due to the high degree of 5-DS disordering.

Because ESR spectra are first derivatives of microwave absorption spectra, the signal intensity of ESR spectra can be estimated by double integration. Calculated signal intensity is proportional to the number of paramagnetic species in a sample. Different samples originally have different numbers of 5-DS molecules. To be able to compare the losses of ESR signal in different samples upon heating, the spectral intensities are divided (normalized) by initial intensity at room temperature. The temperature plots of normalized signal intensity above room temperature have the break point around $330 \mathrm{~K}$ for both onion and tomato embryos (Fig. 2b, adjusted $R^{2}=0.9927$ and
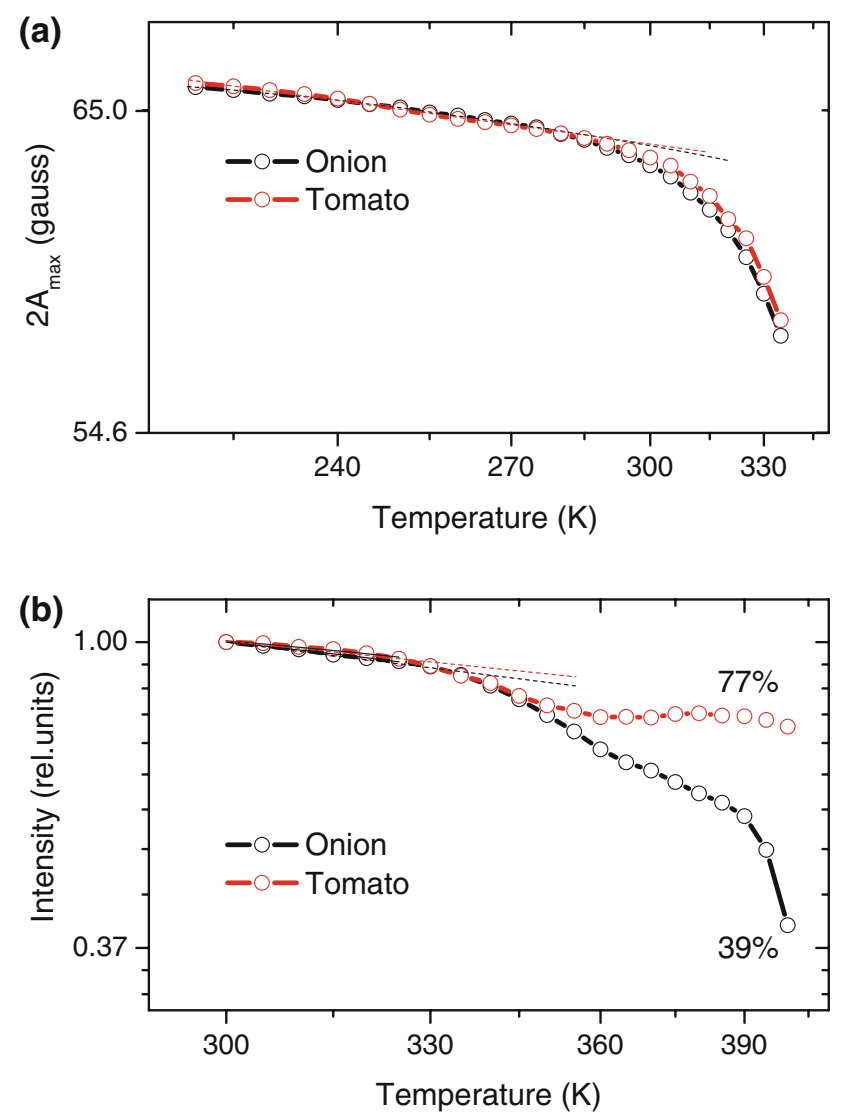

Fig. 2 Temperature plots of $2 \mathrm{~A}_{\max }$ (a) and integrated intensity (b) calculated from 5-DS spectra obtained from labeled dry onion (black) and tomato (red) embryos. The data are plotted in Arrhenius coordinates. The dashed lines represent linear fits. Adjusted $R^{2}$ are indicated in the text
0.99536 for linear fits of onion and tomato embryos, respectively). Further decrease in signal intensity remains similar for both samples up to $345 \mathrm{~K}$. At $T>345 \mathrm{~K}$, the intensity of ESR signal in onion embryos continues to decrease, while remaining almost unchanged in tomato embryos (Fig. 2b).

The reversibility of changes of 5-DS spectra is tested by comparing spectra before heating and immediately after heating, when samples are cooled back to initial (300 K) temperature (Fig. 3a, b). In both samples, a solid-like component of 5-DS spectra disappears upon heating, but appears again upon cooling. The proportion of fluid component is slightly higher after heating/cooling probably because of hysteresis.

The signal intensity of ESR spectra in tomato embryos at $400 \mathrm{~K}$ decreases approximately to $77 \%$ of initial intensity (Fig. 2b); however, after cooling back to room temperature, the signal intensity is almost restored, and finally only $5.9 \%$ of the signal is lost irreversibly (Fig. 3b). In onion embryos, the signal intensity continues to decrease up to $39 \%$ of initial intensity at $400 \mathrm{~K}$ (Fig. 2b). Upon

(a)

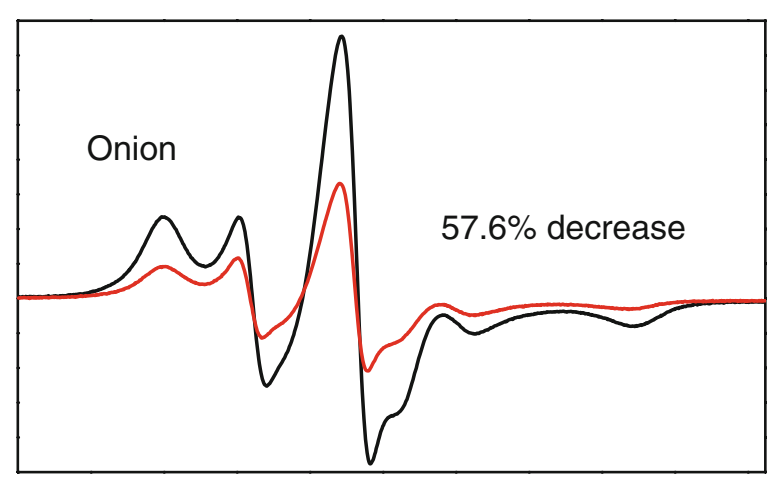

- Before heating After heating

(b)

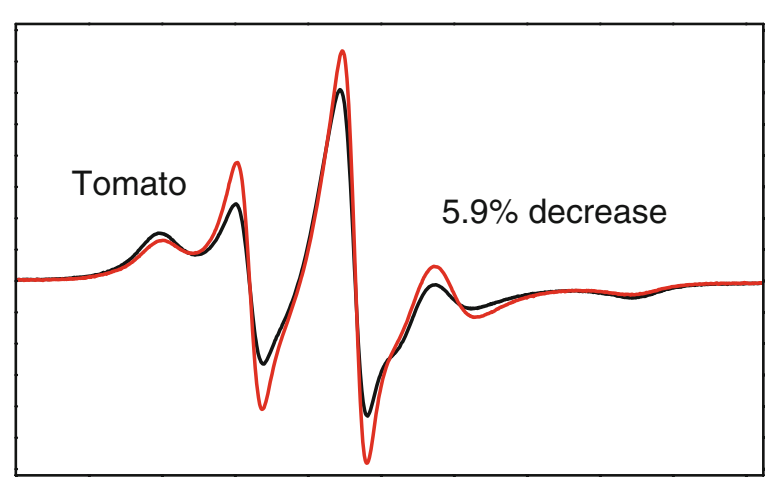

Fig. 3 5-DS spectra from dry onion (a) and tomato (b) embryos before heating to $400 \mathrm{~K}$ (black line) and after heating and cooling down to room temperature (red line). The percentage of not recovered integrated intensity is indicated 
re-cooling, the intensity is only partially restored, so that a considerable part of the signal $(57.6 \%)$ is irreversibly lost (Fig. 3a). We conducted independent experiments with tomato and onion seeds from other seed lots and obtained similar results (data not shown).

The differences in membrane surface chemical stability between onion and tomato seeds appears at $T>345 \mathrm{~K}$ (Fig. 2b). At this temperature, 5-DS are disordered to such an extent that $2 \mathrm{~A}_{\max }$ cannot be determined anymore. Below this temperature, two samples are not different in either ordering of 5-DS (Fig. 2a) or normalized signal intensity (Fig. 2b). At $T>345 \mathrm{~K}$, the fluid-like component dominates in the ESR spectra (Fig. 1). Three relatively narrow lines (Fig. 4a, inset) indicate fast (pseudo) isotropic motion of 5-DS. Dynamic properties of spin labels in the fast motion region are characterized by rotational correlation time $\tau_{\mathrm{c}}$. Although the formula for calculation of $\tau_{\mathrm{c}}$ is valid for isotropic motion (Knowles et al. 1976), we applied it to our system to obtain effective rotational correlation time for 5-DS in melted membranes:

$\tau_{c}=6.5 \times 10^{-10} \Delta W_{0}\left[\operatorname{sqrt}\left(H_{0} / H_{-1}\right)-1\right]$

where $\Delta W_{0}$ is the peak-to-peak width of the central line in gauss, and $H_{0}$ and $H_{-1}$ are the heights of the central and high-field lines, respectively (Fig. 4, inset). Calculated effective $\tau_{\mathrm{c}}$ for the fluid-like component was almost two times higher for 5-DS motion in onion embryos than in tomato ones (Fig. 4).

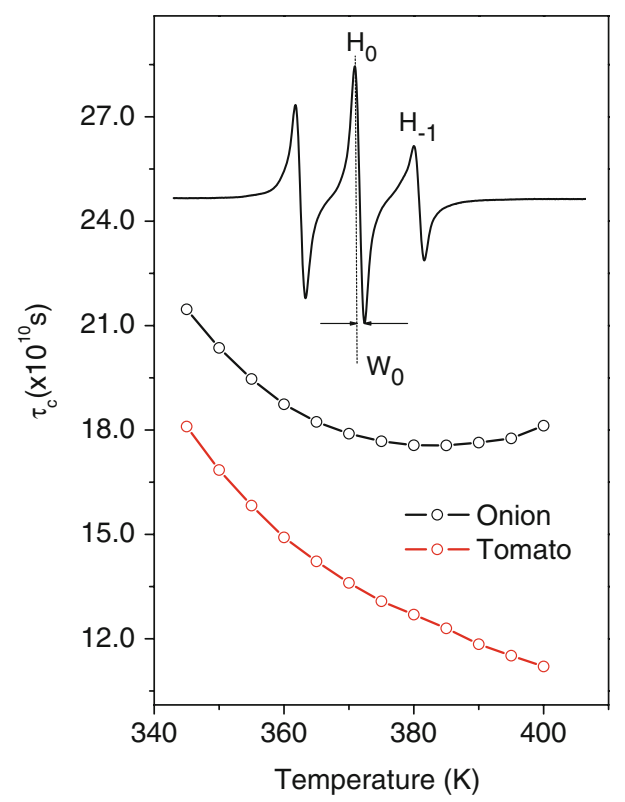

Fig. 4 Temperature plot of rotational correlation time $\tau_{\mathrm{c}}$ calculated from 5-DS spectra of onion (black) and tomato (red) dry embryos at high temperatures. The inset shows 5DS spectrum from onion embryos at $400 \mathrm{~K}$
Dry POPC as a model system

To understand the role of membrane physical state in membrane chemical stability, we conducted model experiments. The model consisted of dry POPC labeled with 5DS. To attribute temperature-induced changes of motional behavior of 5-DS in dry POPC to changes in bilayer physical state, we compared ESR data with temperature plots of vibration frequencies $v$ of different lipid groups obtained from FT-IR experiments.

Figure 5a shows temperature plots of $\nu \mathrm{PO}$ and $\nu \mathrm{CHs}$. Vibration of the phosphate moiety around $1,250 \mathrm{~cm}^{-1} \nu \mathrm{PO}$ is characteristic of a headgroup region (Casal and Mantsch 1984). Symmetric stretching vibration of $\mathrm{CH}$ groups of POPC around 2,850 $\mathrm{cm}^{-1} \nu \mathrm{CHs}$ is used to characterize acyl chain melting (Casal and Mantsch 1984). Both plots have an abrupt upshift in wavenumber around $330 \mathrm{~K}$, which is typical for first-order transitions and indicates cooperative phase transition of the POPC bilayer from gel to liquidcrystalline state. The temperature plot of $v \mathrm{PO}$ also has a

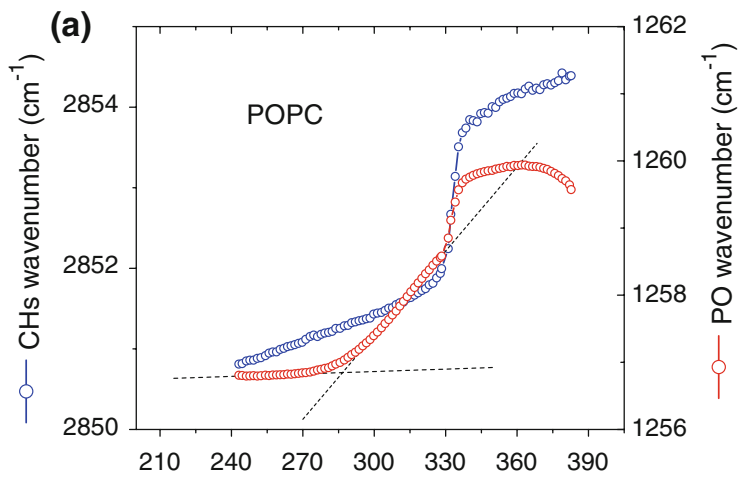

(b)

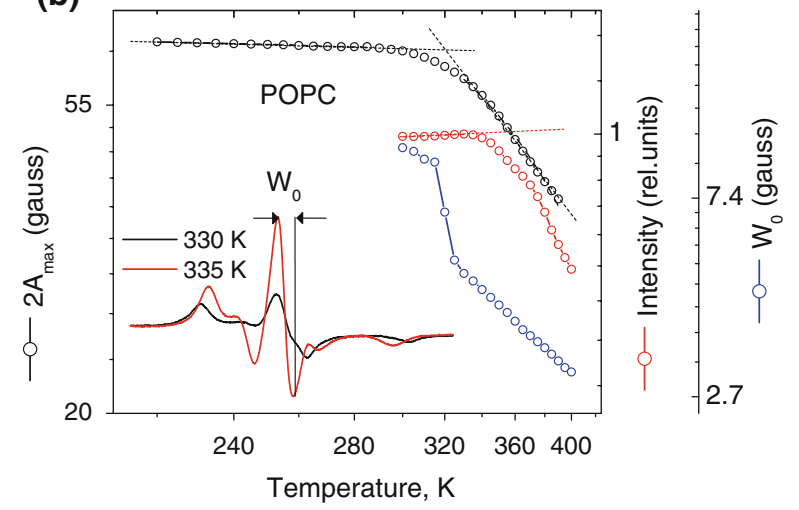

Fig. 5 a Temperature plots of wavenumber $(v)$ position of $\mathrm{CH}$ symmetric (blue) and PO (red) vibrations in dry POPC. b Temperature plots of the distance between the outermost hyperfine extremes $2 \mathrm{~A}_{\max }$ (black), peak-to-peak width of the central line $W_{0}$ (blue), and integrated intensity (red) of 5-DS spectra in dry POPC. The ESR data are plotted in Arrhenius coordinates. The dashed lines represent linear fits. The adjusted $R^{2}$ for all linear fits are indicated in the text. The inset shows 5-DS spectra from dry POPC at 300 and $335 \mathrm{~K}$ 
low-temperature break at $290 \mathrm{~K}$ determined as the interception of two regression lines (Fig. 5a, adjusted $R^{2}=0.90478$ for $T<265 \mathrm{~K}$ and 0.99929 for $295 \mathrm{~K}<T<330 \mathrm{~K}$ ).

The ESR spectra of 5-DS in the model system are onecomponent, and their shape gradually changes with temperature-induced decrease of ordering (Fig. 5b, inset). The temperature dependences of $2 \mathrm{~A}_{\max }$ (as indicated in Fig. 1), the peak-to-peak width of the central line $W_{0}$ (Fig. 5b, inset), and integrated intensity of 5-DS spectra are plotted in Arrhenius coordinates (Fig. 5b). The temperature plot of $W_{0}$ has a sharp downshift at $320 \mathrm{~K}$ (Fig. 5b). It is caused by separation of internal and external hyperfine splittings in ESR spectra at a certain degree of 5-DS disordering (Marsh 1981). The temperature plot of $2 \mathrm{~A}_{\max }$ has only one break point. The interception of two linear regression lines in $2 \mathrm{~A}_{\max }$ plot (adjusted $R^{2}=0.97053$ and 0.99465 for $T<285 \mathrm{~K}$ and $T>335 \mathrm{~K}$, respectively) is found at $315 \mathrm{~K}$. This position is close to the position of the break point in the $W_{0}$ temperature plot but does not coincide with the positions of break points in FT-IR data (Fig. 5a). The breakpoint of ESR signal intensity (adjusted $R^{2}=0.83615$ for linear fit at $T<330 \mathrm{~K}$ ) at $330 \mathrm{~K}$ (Fig. 5b) coincides with $T_{\mathrm{m}}$ of membranes as determined from the $v \mathrm{PO}$ plot (Fig. 5a). There is no sudden change in $2 \mathrm{~A}_{\max }$ and $W_{0}$ at this temperature (Fig. 5b), and the shape of ESR spectrum shows a relatively high degree of immobilization of 5-DS molecules (Fig. 5b, inset).

\section{Dry POPC in sugar matrix}

To separate the role of the membrane physical state and chemical status in membrane chemical stability, POPC dried with trehalose or sucrose sugars was studied. Glucose has a low glass-transition temperature $T_{\mathrm{g}}$ and high reducing potential. Trehalose is a nonreducing sugar with high $T_{\mathrm{g}}$.

Figure 6a shows temperature plots of $\nu \mathrm{PO}$ and $\nu \mathrm{CHs}$ of POPC embedded in the trehalose matrix. Melting of acyl chains is not as sharp as in dry POPC, but the mid-point of transition is at the same temperature as in dry POPC (around $335 \mathrm{~K}$ ). $v \mathrm{PO}$ decreases sharply at $345 \mathrm{~K}<T<370 \mathrm{~K}$ with the mid-point around $360 \mathrm{~K}$. This is the opposite of the sharp increase in $v \mathrm{PO}$ at the membrane phase transition in dry POPC (Fig. 5a). The sharp decrease in $v \mathrm{PO}$ indicates the increase in interactions of PO groups with trehalose during phase transition. Because the melting of the surface of POPC $(v \mathrm{PO})$ in the trehalose matrix occurs at a higher temperature than the melting of acyl chains $(v \mathrm{CHs})$, membrane phase transition cannot be considered as cooperative.

The interception of two regression lines (adjusted $R^{2}=0.96954$ for $T<300 \mathrm{~K}$ and 0.99249 for $T>315 \mathrm{~K}$ ) in the temperature plot of $2 \mathrm{~A}_{\max }$ is found at $309 \mathrm{~K}$ (Fig. 6b). This is slightly higher than the low-temperature
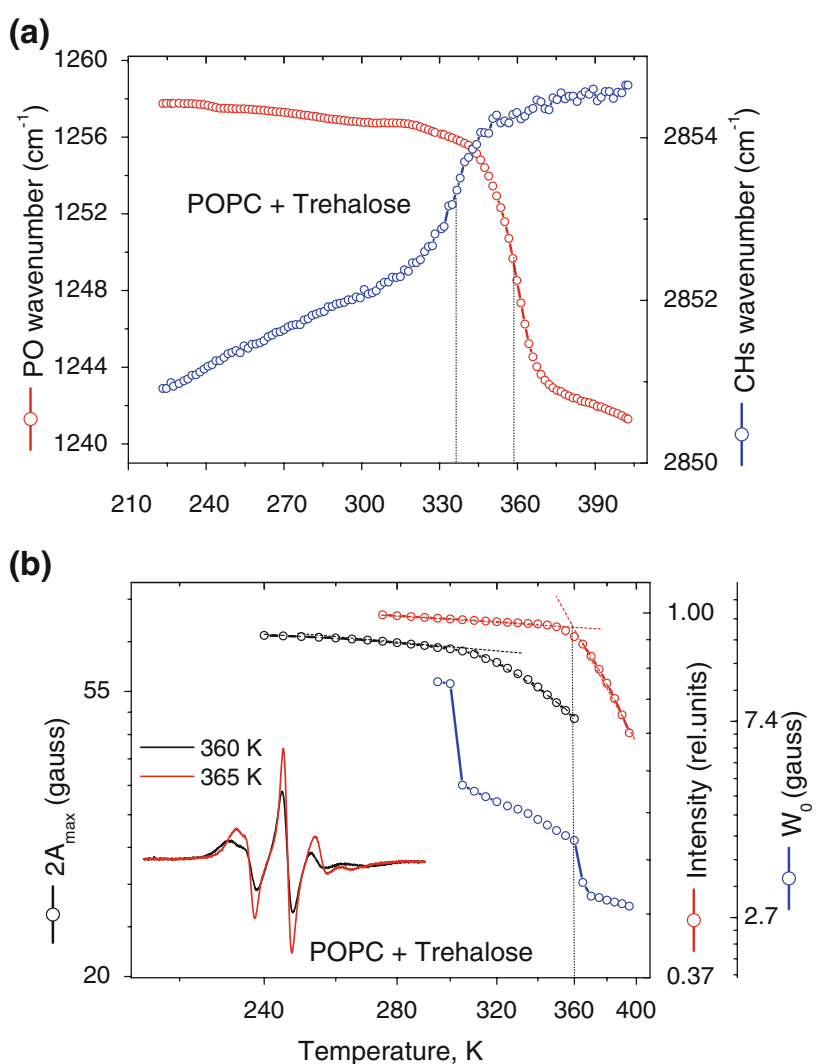

Fig. 6 a Temperature plots of wavenumber $(v)$ position of $\mathrm{CH}$ symmetric (blue) and PO (red) vibrations in dry POPC embedded in trehalose matrix. b Temperature plots of $2 \mathrm{~A}_{\max }$ (black), peak-to-peak width of the central line $W_{0}$ (blue), and integrated intensity (red) of 5-DS spectra in POPC dried with trehalose. The ESR data are plotted in Arrhenius coordinates. The dashed lines represent linear fits. The adjusted $R^{2}$ for all linear fits are indicated in the text. The inset shows 5-DS spectra from dry POPC in trehalose matrix at 360 and $365 \mathrm{~K}$

break of $W_{0}$ (302 K, Fig. 6b). The interception of two regression lines in the signal intensity temperature plot (adjusted $R^{2}=0.96003$ at $T<340 \mathrm{~K}$ and 0.99868 at $T>365 \mathrm{~K}$ ) at $360 \mathrm{~K}$ (Fig. 6b) coincides with the mid-point of the $v$ PO temperature plot (Fig. 6a) and with the hightemperature break of the $W_{0}$ temperature plot (Fig. 6b). The change of the shape of ESR spectra at this temperature also shows the sudden decrease in 5-DS ordering between 360 and $365 \mathrm{~K}$ (Fig. 6b, inset). Thus, in this model, membrane surface melting is accompanied by sudden changes not only in integrated intensity but also in $W_{0}$.

Figure 7a shows the temperature plots of $\nu \mathrm{PO}$ and $\nu \mathrm{CHs}$ for POPC embedded in the glucose matrix. The midpoint of the upshift of $v \mathrm{CHs}$ at around $245 \mathrm{~K}$ indicates acyl chain melting. The decrease in $v \mathrm{PO}$ around $330 \mathrm{~K}$ is not sharp (Fig. 7a). The temperature plot of $2 \mathrm{~A}_{\max }$ has two breakpoints found as interceptions of three linear regression lines (adjusted $R^{2}=0.86696$ at $T<260 \mathrm{~K}, 0.98705$ at $260 \mathrm{~K}<T<310 \mathrm{~K}$, and 0.99787 at $T>330 \mathrm{~K}$ ) (Fig. 7b). The low-temperature break at about $260 \mathrm{~K}$ corresponds to 

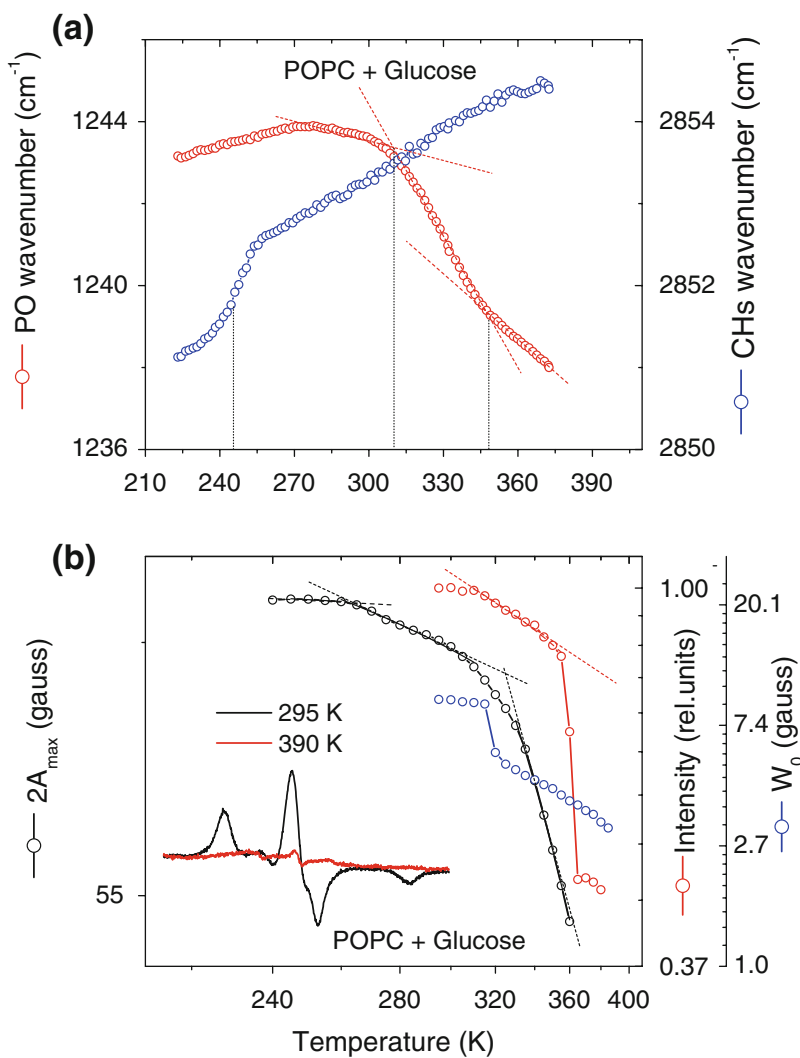

Fig. 7 a Temperature plots of wavenumber position of $\mathrm{CH}$ symmetric (blue) and PO (red) vibrations in dry POPC embedded in glucose matrix. b Temperature plots of $2 \mathrm{~A}_{\max }$ (black), peak-to-peak width of the central line $W_{0}$ (blue), and integrated intensity (red) of 5-DS spectra in POPC dried with glucose. The ESR data are plotted in Arrhenius coordinates. The dashed lines represent linear fits. The adjusted $R^{2}$ for all linear fits are indicated in the text. The inset shows 5 -DS spectra from dry POPC in glucose matrix at 295 and $390 \mathrm{~K}$

the end of acyl chain melting (Fig. 7a). The high-temperature break point at $324 \mathrm{~K}$ is close to the mid-point of the $v$ PO downshift (Fig. 7a). The temperature plot of $W_{0}$ has only one break point at $318 \mathrm{~K}$ (Fig. 7a) and does not correlate with other break points. The signal intensity temperature plot has two break points at 310 and $360 \mathrm{~K}$ (Fig. 7a, adjusted $R^{2}=0.98793$ for the linear fit for $310 \mathrm{~K}<T<355 \mathrm{~K}$ ). The breakpoint at $310 \mathrm{~K}$ coincides with the beginning of the downshift of $\nu \mathrm{PO}$ (Fig. 7a). At $T>360 \mathrm{~K}$, the 5-DS signal was rapidly lost (Fig. 7b) and did not appear upon cooling (data not shown).

\section{5-DS central line intensity}

ESR spectra contain a broad background signal, which originates from other sources than spin label. This signal is slightly visible in ESR spectra only if the signal from the spin label molecules is weak (as in Fig. 7, inset). The background signal has negligible influence on spectral line heights, but its influence on integrated intensity can be considerable. However, it is impossible to use spectral line height for evaluation of the number of paramagnetic centers because it is influenced by line narrowing. The effect of line narrowing can be corrected by calculating line intensity as $W_{0}^{2} \times H_{0}$ (Marsh 1981). The background signal does not influence $W_{0}$. Calculated in such a way, line intensity is proportional to the number of 5-DS molecules in the sample. Figure 8 shows the temperature plots of central line intensities for all three models. In dry POPC, the loss of 5-DS signal is relatively small at $T<370 \mathrm{~K}$ and slightly accelerates at $T>370 \mathrm{~K}$ (adjusted $R^{2}=0.98866$ for linear fit at $325 \mathrm{~K}<T<370 \mathrm{~K}$ ). When POPC is dried with trehalose, slow loss of the 5-DS signal is observed only above $370 \mathrm{~K}$. Glucose causes not only a decrease in 5 -DS signal at lower temperature $(T>320 \mathrm{~K})$, but also its complete and fast disappearance at $T>350 \mathrm{~K}$.

\section{Discussion}

The purpose of this study is to show that membrane surface in long-lived seeds (tomato) is more chemically stable than that in short-lived (onion) seeds. Dry seed embryos were labeled with 5-DS, which partitions into membranes. The reporting doxyl group is located at the membrane surface. The 5-DS spectra above room temperature have two components-solid-like and fluid-like (Fig. 1). Upon heating, $2 \mathrm{~A}_{\max }$ and line heights of the solid-like component decreased together with the increase in line heights of the fluid-like component (Fig. 1). At high temperatures, the fluid-like component dominates the spectra. The changes in the spectral shape are reversible. Therefore the fluid-like

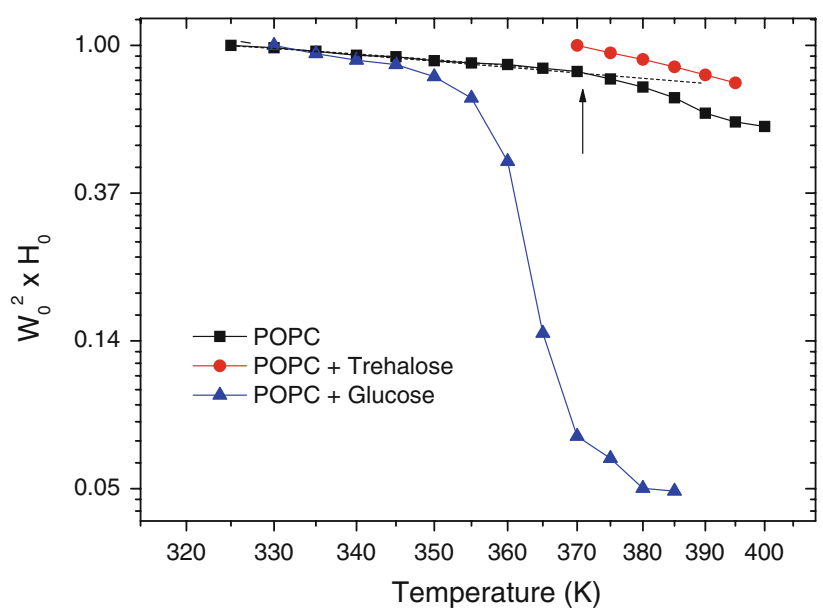

Fig. 8 Temperature plots of normalized (to the maximum value) intensities of the central line of 5-DS spectra, calculated as $W_{0}^{2} \times H_{0}$ at $T>T_{\mathrm{m}}$ of membrane surface for dry POPC (black), dry POPC in trehalose matrix (blue), and dry POPC in glucose matrix (red). The data are plotted in Arrhenius coordinates. The dashed line represents linear fit. The adjusted $R^{2}$ for the linear fit is indicated in the text 
component of 5-DS spectra can be attributed to membrane melting rather than partitioning of spin labels from membranes to oil bodies. The presence of the fluid-like component does not mean that the membrane surface is fluid. 5-DS is the guest molecule, and the increase in the spacing between lipids in the membrane at high temperatures might provide pseudo-isotropic motion of 5-DS.

5-DS spin label is considerably more chemically stable in long-lived tomato embryos than in short-lived onion embryos at $T>345 \mathrm{~K}$ (Fig. 2b). The decrease in the signal intensity of the ESR signal was reversible in tomato seeds and mostly irreversible in onion seeds (Fig. 3a, b). Because the signal intensity in tomato embryos does not change above $345 \mathrm{~K}$ (Fig. 2b), but irreversibly decreases in onion seeds, we assume that all changes in signal intensity above this temperature are irreversible. It is well known that only reduction of nitroxide spin labels to hydroxylamines is reversible. Hydroxylamines can be spontaneously re-oxidized to paramagnetic nitroxides by oxygen (Chen and Swartz 1988). Irreversible loss of 5-DS in dry embryos might relate to oxidation of the spin label moiety because the reduction of oxidized spin label is not probable (Swartz et al. 1986). The spin label also can be reduced to other products than hydroxylamines such as the corresponding amines, and these products cannot be easily re-oxidized (Chen and Swartz 1989). The rate constants of 5-DS reduction/oxidation and re-oxidation of corresponding hydroxylamines in dry systems are diffusion-dependent and therefore temperature-dependent. Probably, the reversible shift of the steady-state concentrations of 5-DS at $T<345 \mathrm{~K}$ in tomato embryos is caused by spin label reduction to hydroxylamines. It is known that the reduction of the spin labels to hydroxylamines is slower at low temperatures (Morse 1985). Irreversible loss of 5-DS signal at $T>345 \mathrm{~K}$ in onion embryos might relate to 5-DS oxidation or reduction to products other than hydroxylamines.

Because rate constants of spin label reduction/oxidation are diffusion-dependent, it is reasonable to expect relationships between molecular ordering of 5-DS and the chemical stability of the signal. However, we did not find any differences in the temperature plots of $2 \mathrm{~A}_{\max }$ for tomato and onion embryos (Fig. 2a). Nor were any differences found between normalized signal intensities for the two species below $345 \mathrm{~K}$. Above $345 \mathrm{~K}$, the spectra show mainly pseudo-isotropic motion of 5-DS, which can be characterized by the effective rotational correlation time $\tau_{\mathrm{c}}$. The temperature plots of $\tau_{\mathrm{c}}$ show that the rotation of the spin label moiety is faster in the melted membrane phase of long-lived tomato embryos (Fig. 4). Therefore, the slower rotation of the spin probe molecules does not correlate with better chemical stability of 5-DS.

The different character of motional and chemical parameters might be one of the reasons why correlation between motion behavior and chemical stability of 5-DS in seed embryos was not found. Seed embryos are heterogeneous systems. The shape of 5-DS spectrum obtained from labeled embryos is the result of superposition of many spectra originating from different locations of 5-DS molecules. Therefore, the resulting $2 \mathrm{~A}_{\max }$ is an average (effective) parameter. In contrast to $2 \mathrm{~A}_{\max }$, the integrated intensity of spectra is a cumulative parameter. All the changes in signals originating from different locations are summed up in the integrated intensity of spectra. In highly heterogeneous systems as seed embryos, reduction/oxidation of 5-DS might occur in limited numbers of locations. The motional characteristics of 5-DS from these locations might not be visible because of averaging out. Therefore, relationships between 5-DS immobilization and chemical stability should be studied on homogeneous systems.

The lack of correlation between motion parameters and chemical stability of 5-DS in in vivo experiments might also indicate that in these seeds the differences in membrane chemical stability are caused by differences in the chemical status of the membrane environment rather than in the degree of membrane immobilization. The model experiments are conducted to separate the roles of membrane physical state and chemical status in 5-DS chemical conversion to nonparamagnetic species.

Another advantage of using model system is the possibility to use FT-IR spectroscopy together with spin-label technique. 5-DS is a guest molecule. Spin label motion depends on the size of the cage where it resides and is not always coupled with the behavior of the environment. However, spin label technique is more suitable for in vivo study of membrane dynamics than FT-IR. The FT-IR study of membrane acyl chain melting cannot be carried out on seeds that contain oil (Sacande et al. 2001).

5-DS-labeled dry bilayer was used as a model membrane. The physical state of the bilayer was estimated by FT-IR from the temperature plots of $v \mathrm{PO}$ (head-group area) and $v$ CHs (acyl chains). 5-DS spectra in dry POPC have only one component (Fig. 5b, inset) and this simplifies the analysis. The decrease in membrane ordering with temperature is estimated from the temperature plots of the outermost hyperfine extremes $2 \mathrm{~A}_{\max }$. The temperature plots of the peak-to-peak width of the central line $W_{0}$ provide information about the rate of rotation without respect to the degree of spin label ordering.

In dry POPC membrane, phase transition is found at $330 \mathrm{~K}$ as cooperative melting of both acyl chains ( $v \mathrm{CHs}$ ) and bilayer surface ( $v \mathrm{PO})$ (Fig. 5a). The temperature plot of $v \mathrm{PO}$ also shows the second-order-like transition at $290 \mathrm{~K}$. This type of transition has been observed in the temperature plots of $\mathrm{OH}$ vibrations in sugar glasses and has been attributed to glass transition (Wolkers and Hoekstra 2003). The nature of the low-temperature break in $v \mathrm{PO}$ is 
not known. We can consider it as a sign of some structural changes that take place at the membrane surface in the dry state before membrane melting. The existence of pretransition and sub-transition in hydrated membranes is well known (Heimburg 2000; Smeller and Györgyi 1989). Subtransition is associated with rotational disordering of the acyl chains (Le Bihan and Pezolet 1998). Pretransition is linked to the formation of periodic ripples on the membrane surface (Heimburg 2000). Ripples cannot be observed in dry membranes. Therefore, similarities with subtransition (disordering) are more probable. Shalaev and Steponkus (2003) showed the presence of glass transition of the $\mathrm{L}_{\beta}$ phase of POPE and related it to several different types of molecular motion. Voinova (1996) presented the theory of membrane vitrification. However, the exact nature of structural changes at the membrane surface below $T_{\mathrm{m}}$ is still not understood.

In dry POPC, the low-temperature break of $v \mathrm{PO}$ and the break of $2 \mathrm{~A}_{\max }$ do not precisely match each other (Fig. 5a, b). However, the temperature plot of $2 \mathrm{~A}_{\max }$ below this break can be fitted with a straight line, which is characteristic of libration motion of nitroxides in a glassy state (Dzuba 2000). Moreover, it was shown (Dzuba et al. 1993) that the small nitroxide Tempone experiences libration motion in dry soybean phospholipid mixture at $77 \mathrm{~K}$. All data taken together allow the assumption of the existence of a glassy state at the surface of dry membranes. However, the data in this study are not enough to attribute the break in $2 \mathrm{~A}_{\max }$ of 5-DS spectra to the glass transition at the membrane surface. Whatever the nature of the temperature-induced structural changes in the head group area of dry POPC below $T_{\mathrm{m}}$ in our models (Fig. 5a), these changes cause a decrease in molecular ordering of 5-DS, but do not influence membrane chemical stability (Fig. 5b).

When POPC was dried in the presence of non-reducing trehalose, the mid-point of the upshift of $v \mathrm{CHs}$ (acyl chains) was observed around $335 \mathrm{~K}$, while bilayer surface melting ( $v \mathrm{PO}$ ) was found at $360 \mathrm{~K}$ (Fig. 6a). The different melting temperatures for acyl chains and head-group area indicate that membrane melting is not cooperative in this model. Even more differences in the temperature of acyl chains $\left(T_{\mathrm{m}}=245 \mathrm{~K}\right)$ and head group $\left(T_{\mathrm{m}}=330 \mathrm{~K}\right)$ melting were observed when POPC was dried in the presence of glucose (Fig. 7a). The noncooperative membrane melting is probably caused by interactions of sugars with POPC. The considerable temperature shift of $T_{\mathrm{m}}$ of acyl chain (from 335 to $245 \mathrm{~K}$ ) in POPC dried with glucose in comparison with dry POPC without sugar (Figs. 5a, 7a) unambiguously shows the interactions of glucose with POPC molecules. The interactions of trehalose with POPC are not so obvious because the presence of trehalose does not shift the $T_{\mathrm{m}}$ of acyl chains (Fig. 6a). However, the increase in $T_{\mathrm{m}}$ for the $v \mathrm{PO}$ from 335 to $360 \mathrm{~K}$ shows that trehalose interacts with POPC molecules. Probably in this case, the interactions are shallow and cause immobilization of membrane surface without increasing spacing between lipids. The sharp decrease in the vibrational frequency of $\mathrm{PO}$ around $360 \mathrm{~K}$ for POPC in the trehalose matrix and around $330 \mathrm{~K}$ for POPC in the glucose matrix (Fig. 6a, 7a) indicates the increase in interactions between $\mathrm{PO}$ groups of lipids and sugars during bilayer surface melting. A similar sharp downshift in CO vibration frequencies in hydrated DPPC has been interpreted as increasing interactions between carbonyl groups and water during membrane phase transition (Schwieger and Blume 2007). The decrease in $T_{\mathrm{m}}$ of acyl chains during the second heating cycle of dry POPC in trehalose matrix supports this interpretation (data not shown).

Melting of membrane surface in the models with sugars correlates with the disappearance of the ESR signal (Figs. 6, 7). However, the extent and reversibility of the signal loss were different for POPC embedded in glucose and trehalose matrixes. When POPC was dried in glucose, the loss of the signal was complete (Fig. 7b inset) and irreversible (data not shown). To exclude the role of the temperature changes of the background signal of the ESR spectra, the intensity of the central line $\left(W_{0}^{2} \times H_{0}\right)$ of 5-DS spectra was plotted against the temperature for all three models above $T_{\mathrm{m}}$ of bilayer surface (Fig. 8). The decrease in the intensity of the central line of 5-DS was observed in all models (Fig. 8). This confirms that the decrease in the integrated intensity of ESR spectra in Figs. 5-7 results from chemical conversion of 5-DS molecules and not from the changes of background.

The data obtained on model systems allow separation of the roles of the physical state of the membranes and the chemical status of the environment in membrane chemical stability. Because the beginning of chemical conversion of the 5-DS molecules to nonparamagnetic species coincides with membrane surface melting, the degree of membrane surface immobilization determines the temperature above which chemical reactions become possible. However, the extent of chemical reactions and their reversibility are determined by the chemical status of the nearby environment. Based on the results of the model experiments, we suggest that the effective (averaged over the sample) temperature of membrane surface melting is around $330 \mathrm{~K}$ for both onion and tomato embryos. The fast and irreversible disappearance of the spin label signal in onion seeds at $T>345 \mathrm{~K}$ might be caused by the presence of reducing/oxidizing agents that chemically and irreversibly convert 5-DS to nonparamagnetic species. Therefore, the short longevity of onion seeds in comparison with tomato seeds might be caused by their chemical status rather than the degree of membrane immobilization. 
In conclusion, the data presented in this study show that chemical thermostability of the membrane surface contains valuable information that can be used to predict storage behavior of seeds. However, the proposed approach needs further detailed investigation.

Acknowledgments This work was supported by CW/ALW grant no. 805.49.001 from the Dutch Organization for Scientific Research (NWO) and by project no. 10195 from the Dutch Foundation for Technological Research STW. The authors thank the Centre for Genetic Resources, the Netherlands (CGN), for providing onion and tomato seeds for the experiments.

Open Access This article is distributed under the terms of the Creative Commons Attribution Noncommercial License which permits any noncommercial use, distribution, and reproduction in any medium, provided the original author(s) and source are credited.

\section{References}

Bailly C (2004) Active oxygen species and antioxidants in seed biology. Seed Sci Res 14:93-107

Beckman KB, Ames BN (1997) Oxidants, antioxidants, and aging. In: Scandalios JG (ed) Oxidative stress and the molecular biology of antioxidant defenses. Cold Spring Harbor Laboratory Press, Plainview, NY

Benson EE (1990) Free radical damage in stored plant germplasm. International Board of Plant Genetic Resources (IBPGR), Rome

Bobko AA, Kirilyuk IA, Grigorév IA, Zweier JL, Khramtsov VV (2007) Reversible reduction of nitroxides to hydroxylamines: roles for ascorbate and glutathione. Free Radic Biol Med 42:404-412

Buitink J, Claessens MMAE, Hemminga MA, Hoekstra FA (1998) Influence of water content and temperature on molecular mobility and intracellular glasses in seeds and pollen. Plant Physiol 118:531-541

Buitink J, Leprince O, Hemminga MA, Hoekstra FA (2000) Molecular mobility in the cytoplasm: an approach to describe and predict lifespan of dry germplasm. Proc Natl Acad Sci USA 97:2385-2390

Buitink J, Hoekstra FA, Leprince O (2002) Biochemistry and biophysics of tolerance systems. In: Black M, Pritchard HW (eds) Desiccation and survival in plants: drying without dying. CAB International/Wallingford, Oxon, UK, pp 293-318

Casal HL, Mantsch HH (1984) Polymorphic phase behavior of phospholipid membranes studied by infrared spectroscopy. Biochim Biophys Acta 779:381-401

Chen K, Swartz HM (1988) Oxidation of hydroxylamines to nitroxide spin labels in living cells. Biochim Biophys Acta 970:270-277

Chen K, Swartz HM (1989) The products of the reduction of doxyl stearates in cells are hydroxylamines as shown by oxidation by 15N-perdeuterated tempone. Biochim Biophys Acta 992:131133

Chen K, Morse PD, Swartz NM (1988) Kinetics of enzyme-mediated reduction of lipid soluble nitroxide spin labels by living cells. Biochim Biophys Acta 943:477-484

Chen K, Glockner JF, Morse PD II, Swartz NM (1989) Effects of oxygen on the metabolism of nitroxide spin labels in cells. Biochemistry 28:2496-2501

Copeland LO, McDonald MB (2001) Principles of seed science and technology. Lower Academic Publishers, Dordrecht
Delouche JC, Baskin CC (1973) Accelerated aging techniques for predicting the relative storability of seed lots. Seed Sci Technol $1: 427-452$

Dzuba SA (2000) Libration motion of guest spin probe molecules in organic glasses: CW EPR and electron spin echo study. Spectrochim Acta A 56:227-234

Dzuba SA, Golovina YA, Tsvetkov YD (1993) Echo-induced EPR spectra of spin probes as a method for identification of glassy states in biological objects. J Magn Reson B 101:134-138

Ellis RH, Roberts EH (1980) Improved equations for the prediction of seed longevity. Ann Bot 45:13-30

Hay FR, Mead A, Manger K, Wilson FJ (2003) One-step analysis of seed storage data and the longevity of Arabidopsis thaliana seeds. J Exp Bot 54:993-1011

Heimburg T (2000) A model for the lipid pretransition: coupling of ripple formation with the chain-melting transition. Biophys $\mathrm{J}$ 78:1154-1165

Hendry GAF (1993) Oxygen, free radical processes and seed longevity. Seed Sci Res 3:141-153

Hoekstra FA (2005) Differential longevities in desiccated anhydrobiotic plant systems. Integr Comp Biol 45:725-733

Kaplan J, Canonico PG, Caspary WJ (1973) Electron spin resonance studies of spin-labeled mammalian cells by detection of surface membrane signals. Proc Natl Acad Sci USA 70:66-70

Knowles PF, Marsh D, Rattle HWE (1976) Magnetic resonance of biomolecules: an introduction to the theory of NMR and ESR in biological systems. Wiley, London

Kocherginsky N, Swartz HM, Sentjurc M (1995) Nitroxide spin labels: reactions in biology and chemistry. CRC Press, Boca Raton

Le Bihan T, Pezolet M (1998) Study of the structure and phase behavior of dipalmitoylphosphatidylcholine by infrared spectroscopy: characterization of the pretransition and subtransition. Chem Phys Lipids 94:13-33

Leopold AC, Sun WQ, Bernal-Lugo I (1994) The glassy state in seeds: analysis and function. Seed Sci Res 4:267-274

Marsh D (1981) Electron spin resonance: spin labels. Mol Biol Biochem Biophys 31:51-142

Morse PD (1985) Structure-function relationships in cell membranes as revealed by spin labeling EPR. In: Benga G (ed) Structure and properties of cell membranes, vol 3. CRC Press, Boca Raton, pp $195-236$

Narayana Murthy UM, Kumar PP, Sun WQ (2003) Mechanisms of seed ageing under different storage conditions for Vigna radiata (L) Wilczek: lipid peroxidation, sugar hydrolysis, Maillard reactions and their relationship to glass state transition. J Exp Bot 54:1057-1067

Priestley DA (1986) Seed aging: implications for seed storage and persistence in soil. Comstock, Ithaca, NY

Priestley DA, Cullinan VI, Wolfe J (1985) Differences in seed longevity at the species level. Plant Cell Environ 8:557-562

Roberts EH (1973) Prediction the storage life of seeds. Seed Sci Technol 1:499-514

Sacande M, Golovina EA, van Aelst AC, Hoekstra FA (2001) Viability loss of neem (Azadirachta indica) seeds associated with membrane phase behaviour. J Exp Bot 52:919-931

Schwieger C, Blume A (2007) Interaction of poly(L-lysines) with negatively charged membranes: an FT-IR and DSC study. Eur Biophys J 36:437-450

Sentjurc M, Bacic G, Swartz HM (1990) Reduction of doxyl stearates by ascorbate in unilamellar liposomes. Arch Biochem Biophys 282:207-213

Shalaev EY, Steponkus PL (2003) Glass transition of a synthetic phospholipid in the lamellar phase. J Phys Chem B 107:8734-8737

Smeller L, Györgyi S (1989) A statistical mechanical model of the pre- and subtransitions of lecithin membranes. J Theor Biol 137:203-214 
Stier A, Reitz I (1971) Radical production in amine oxidation by liver microsomes. Xenobiotica 1:499-500

Stier A, Sackmann E (1973) Spin labels as enzyme substrates. Heterogeneous lipid distribution in liver microsomal membranes. Biochim Biophys Acta 311:400-408

Sun WQ (1997) Glassy state and seed storage stability: the WLF kinetics of seed viability loss at $T>T_{\mathrm{g}}$ and the plasticization effect of water on storage stability. Ann Bot 79:291-297

Swartz HM (1987) Use of nitroxides to measure redox metabolism in cells and tissues. J Chem Soc Faraday Trans 83(1):191-202

Swartz HM (1990) Principles of the metabolism of nitroxides and their implications for spin trapping. Free Radic Res Commun 9:399-405

Swartz HM, Sentjurc M, Morse PD (1986) Cellular metabolism of water-soluble nitroxides: effect on rate of reduction of cell/ nitroxide ratio, oxygen concentrations, and permeability of nitroxides. Biochim Biophys Acta 888:82-90

Tang S, TeKrony DM, Egli DB, Cornelius PL (2000) An alternative model to predict corn seed deterioration during storage. Crop Sci 40:463-470

Voinova MV (1996) The theory of membrane "vitrification". Thermochim Acta 280(281):465-477

Wilson DO, McDonald MB (1986) The lipid peroxidation model of seed aging. Seed Sci Technol 14:269-300

Wolkers WF, Hoekstra FA (2003) In situ FTIR assessment of desiccation-tolerant tissues. Spectroscopy 17:297-313

Wolkers WF, Oldenhof H, Alberda M, Hoekstra FA (1998) A Fourier transform infrared microspectroscopy study of sugar glasses: application to anhydrobiotic plant cells. Biochim Biophys Acta 1379:83-96 